\title{
GTSE1 promotes SNAIL1 degradation by facilitating its nuclear export in hepatocellular carcin oma cells
}

\author{
SHAN-SHAN LI ${ }^{1,2^{*}}$, DONG-MEI CHEN ${ }^{1 *}$, LU-BIAO CHEN $^{1}$, HUAN WEI $^{1}$, JIA-LIANG WANG $^{1}$, \\ JIAN XIAO ${ }^{2}$, YUE-HUA HUANG ${ }^{1,3}$ and YI-FAN LIAN ${ }^{1}$
}

${ }^{1}$ Guangdong Provincial Key Laboratory of Liver Disease Research, The Third Affiliated Hospital of Sun Yat-sen University, Guangzhou, Guangdong 510630; ${ }^{2}$ Department of Medical Oncology, The Sixth Affiliated Hospital of Sun Yat-sen University, Guangzhou, Guangdong 510655; ${ }^{3}$ Department of Infectious Diseases, The Third Affiliated Hospital of Sun Yat-sen University, Guangzhou, Guangdong 510630, P.R. China

Received August 18, 2020; Accepted February 2, 2021

DOI: $10.3892 / \mathrm{mmr} .2021 .12093$

\begin{abstract}
Snail family transcriptional repressor 1 (SNAIL1) is a master inducer of the epithelial-to-mesenchymal transition (EMT) process, contributing to tumor metastasis and recurrence. Our previous study reported that $\mathrm{G}_{2}$ and $S$ phase-expressed-1 (GTSE1) served a role in regulating SNAIL1 expression in hepatocellular carcinoma (HCC). However, the underlying mechanism remains unknown. Therefore, the present study aimed to reveal the regulatory mechanism of GTSE1 on SNAIL1 expression using in vitro assays performed in HCC cell models. It was demonstrated that endogenous SNAIL1 expression was downregulated and upregulated by GTSE1 overexpression or small interfering RNA-mediated knockdown, respectively. Via cycloheximide chase experiments, it was identified that GTSE1 overexpression increased the protein turnover of SNAIL1, while knockdown of GTSE1 reduced its degradation rate. Furthermore, it was demonstrated that GTSE1 overexpression induced the cytoplasmic expression of SNAIL1 using immunofluorescence and subcellular fractionation methods. The nuclear export inhibitor leptomycin B was able to decrease the cytoplasmic retention of
\end{abstract}

Correspondence to: Dr Yi-Fan Lian or Dr Yue-Hua Huang, Guangdong Provincial Key Laboratory of Liver Disease Research, The Third Affiliated Hospital of Sun Yat-sen University, 600 Tian He Road, Guangzhou, Guangdong 510630, P.R. China

E-mail: lianyf@mail3.sysu.edu.cn

E-mail: huangyh53@mail.sysu.edu.cn

${ }^{*}$ Contributed equally

Abbreviations: CHX, cycloheximide; EMT, epithelial-tomesenchymal transition; GTSE1, $\mathrm{G}_{2}$ and $\mathrm{S}$ phase-expressed-1; HCC, hepatocellular carcinoma; LMB, leptomycin B; RT-qPCR, reverse transcription-quantitative PCR; SBEs, Smad binding elements; TSS, transcription start site; WB, western blotting

Key words: $\mathrm{G}_{2}$ and $\mathrm{S}$ phase-expressed-1, snail family transcriptional repressor 1 , hepatocellular carcinoma, TGF- $\beta /$ Smad signaling
SNAIL1 caused by GTSE1 overexpression. In addition, TGF- $\beta$ I treatment increased both the mRNA and protein expression levels of GTSE1, and decreased the protein expression level of SNAIL1 without affecting its mRNA transcription in Huh7 cells. It was also found that TGF- $\beta$ signaling could upregulate the transcription of GTSE1 expression by transactivating the Smad binding elements in the GTSE1 promoter. Moreover, the TGF- $\beta$ I-induced decrease in SNAIL1 protein expression was GTSE1-dependent in Huh7 cells. In conclusion, the current study provides a novel mechanism via which GTSE1 affects the stability of SNAIL1 by regulating its subcellular localization in HCC cells.

\section{Introduction}

Hepatocellular carcinoma (HCC) is the most common type of malignant tumor in the liver, leading to $\sim 750,000$ deaths annually worldwide (1). Despite progress in the HCC treatment options, including liver transplantation, surgery and targeted therapy, the 5-year survival rate of patients with HCC has remained low, partially due to tumor metastasis and recurrence $(2,3)$. Therefore, enhanced understanding of the molecular mechanisms underlying HCC development and progression, especially those associated with tumor metastasis and recurrence, is essential for improving the prognosis and treatment of patients with HCC.

Snail family transcriptional repressor 1 (SNAIL1) is a famous transcriptional repressor that uses its C-terminal zinc finger domain to bind to the E-box motif of the target gene promoters and its evolutionarily conserved N-terminal Snail/Gfi domain to recruit other transcriptional corepressor complexes (4). The central region of SNAIL1, containing a serine-rich domain and a nuclear export sequence, is important for the regulation of its protein stability and subcellular localization, respectively (5). As a critical regulator of epithelial-to-mesenchymal transition (EMT), a molecular program promoting the metastatic cascade of cancer cells, SNAIL1 expression has been reported to be closely associated with the metastasis of multiple solid tumors, including HCC (6). Moreover, SNAIL1 has been associated with tumor recurrence based on its facilitation of cancer stem cell generation and 
resistance to chemo- and radiotherapy (7). SNAIL1 has been shown to be sufficient to promote mammary tumor recurrence in vivo, and a high level of SNAIL1 is associated with decreased relapse-free survival rates (8). Therefore, SNAIL1 could be an attractive target for preventing tumor metastasis and recurrence.

SNAIL1 expression is modulated by various signals at the transcriptional and post-translational levels. For example, numerous soluble factors, including TGF- $\beta$, regulate SNAIL1 mRNA transcription by activating downstream transcription factors, such as Smads (9). Protein phosphorylation, ubiquitination or O-linked $\beta$-N-acetylglucosamine can exert differential regulatory effects on the localization, stability or transcriptional activity of SNAIL1, dependent on the functional protein and subsequent signaling events (10). Moreover, SNAIL1 is a labile protein whose subcellular localization has an important effect on its stability. SNAIL1 exerts its transcriptional regulatory activity in the nucleus, where its turnover is slow, while in the cytosol, it is rapidly degraded by proteasomes $(11,12)$.

Our previous study reported that $\mathrm{G}_{2}$ and $\mathrm{S}$ phase-expressed-1 (GTSE1) was involved in regulating the expression level of SNAIL1 protein and promoted the metastatic ability of HCC cells via the EMT process (13). GTSE1 can also negatively regulate $\mathrm{p} 53$ function by stimulating the cytoplasmic localization of p53 and downregulating its protein levels (14). Moreover, GTSE1 was found to be associated with increased invasive potential by interaction with microtubule plus-end binding protein EB1 in breast cancer cells (15). In addition, upregulation of GTSE1 has been observed in liver and lung cancer types, and could function to inhibit the apoptotic signaling and induce chemoresistance in gastric cancer (16).

Since SNAIL1 serves a vital role in tumor metastasis and recurrence, the current study investigated the exact mechanism via which the multifunctional protein GTSE1 regulates SNAIL1 expression in HCC cells.

\section{Materials and methods}

Reagents. Small molecule inhibitors were as follows: MG-132 (cat. no. 474790; EMD Millipore), cycloheximide (CHX; cat. no. C7698, Sigma-Aldrich; Merck KGaA) and leptomycin B (LMB; cat. no. HY-16909; MedChemExpress). TGF- $\beta$ I was purchased from PeproTech, Inc. (cat. no. 100-21-2). Briefly, MG-132 was used at a concentration of $10 \mu \mathrm{M}$ for $12 \mathrm{~h}$ at $37^{\circ} \mathrm{C}$; LMB was used at a concentration of $50 \mathrm{ng} / \mathrm{ml}$ for $12 \mathrm{~h}$ at $37^{\circ} \mathrm{C}$; TGF- $\beta$ I was used at a concentration of $20 \mathrm{ng} / \mathrm{ml}$ for $24 \mathrm{~h}$ at $37^{\circ} \mathrm{C}$ in Huh7 cells. All other chemical reagents were obtained from Sigma-Aldrich (Merck KGaA), unless otherwise indicated.

Cell culture. This study employed 293T cells and two hepatocellular carcinoma cell lines, Huh7 and PLC/PRF/5. All cell lines were obtained from the CellCookBiotech Co., Ltd. Mycoplasma testing was performed for all the cell lines used, which were authenticated by short tandem repeat analysis. Cells were cultured in DMEM (Invitrogen; Thermo Fisher Scientific, Inc.) containing 10\% FBS (Gibco; Thermo Fisher Scientific, Inc.) at $37^{\circ} \mathrm{C}$ and $5 \% \mathrm{CO}_{2}$. Cells were digested and passaged as previously described (17), passage 3-7 (early passages) were used for assays.
Transwell assay. Cells were trypsinized and pelleted via centrifugation at a speed of $400 \mathrm{x} \mathrm{g}$ for $5 \mathrm{~min}$ at room temperature. After washing twice in 1X PBS, Huh7 cells were resuspended in serum-free DMEM at a density of $5 \times 10^{5}$ cells $/ \mathrm{ml}$, and $200 \mu \mathrm{l}$ cell suspension was seeded onto the upper chambers of the Transwell inserts (pore size, $8 \mu \mathrm{m}$; cat. no. 3422; Corning). DMEM containing 10\% FBS was added to the lower chamber, FBS served as a chemoattractant. After incubation at $37^{\circ} \mathrm{C}$ for $20 \mathrm{~h}$, the non-migrated cells were gently removed with a cotton swab. Migrated cells located on the lower side of the chamber were fixed with $4 \%$ paraformaldehyde for $20 \mathrm{~min}$ at room temperature prior to crystal violet (cat. no. C0121; Beyotime Institute of Biotechnology) staining for $1 \mathrm{~h}$ at room temperature. The number of migrated cells was examined via light microscopic observation at x200 magnification (DMi8; Leica Microsystems, Inc.).

Cell proliferation assay. The cell proliferative rate was determined using a Cell Counting Kit-8 (CCK-8) assay (cat. no. CK04; Dojindo Molecular Technologies, Inc.), according to the manufacturer's protocol. In brief, Huh7 cells were seeded in five replicates in a 96-well plate at a density of 1,000 cells and cultured with $100 \mu \mathrm{l}$ DMEM containing 10\% FBS per well. For measurement, $10 \mu$ l CCK- 8 solution was added to each well, and the cells were incubated for another $4 \mathrm{~h}$ at $37^{\circ} \mathrm{C}$. Viable cells were counted every day for a period of 5 days by reading the absorbance at $450 \mathrm{~nm}$ with a plate reader (ELx800; BioTek Instruments, Inc.).

Cell cycle analysis. In brief, $5 \times 10^{5}$ Huh7 cells were harvested in fresh medium. Samples were washed in 1X PBS, and then fixed in ice-cold $70 \%$ ethanol at $-20^{\circ} \mathrm{C}$ overnight. Fixed cells were washed with cold 1X PBS and stained with FxCycle ${ }^{\mathrm{TM}}$ PI/RNase staining solution (cat. no. F10797; Invitrogen; Thermo Fisher Scientific, Inc.) for $30 \mathrm{~min}$ at room temperature in the dark. Cells were then analyzed using a BD LSR II ${ }^{\mathrm{TM}}$ flow cytometry (BD Biosciences), and data were analyzed with FlowJo software (version 10; FlowJo LLC).

Reverse transcription-quantitative PCR (RT-qPCR). Total RNA was isolated from 293T or Huh7 cells using TRIzol ${ }^{\circledR}$ reagent (Invitrogen; Thermo Fisher Scientific, Inc.) according to the manufacturer's protocol. Total RNA $(1 \mu \mathrm{g})$ was reverse transcribed at $42^{\circ} \mathrm{C}$ for $1 \mathrm{~h}$ into cDNA using the GoScript ${ }^{\mathrm{TM}}$ Reverse Transcription system (cat. no. A5002; Promega Corporation) according to the manufacturer's protocol. PCR was performed with Platinum SYBR-Green qPCR SuperMix-UDG (Invitrogen; Thermo Fisher Scientific, Inc.) with a LightCycler 480 PCR platform (Roche Diagnostics). The thermocycling conditions were as follows: Initial denaturation for $10 \mathrm{~min}$ at $95^{\circ} \mathrm{C}, 40$ cycles of denaturation for $15 \mathrm{sec}$ at $95^{\circ} \mathrm{C}$, annealing for $40 \mathrm{sec}$ at $55^{\circ} \mathrm{C}$ and elongation for $20 \mathrm{sec}$ at $72^{\circ} \mathrm{C}$, followed by final extension for $3 \mathrm{~min}$ at $72^{\circ} \mathrm{C}$. Specific primers were as follows: GTSE1 forward, 5'-CAGGGGACGTGAACATGGATG-3' and reverse, 5'-ATG TCCAAAGGGTCCGAAGAA-3'; SNAIL1 forward, 5'-TCG GAAGCCTAACTACAGCGA-3' and reverse, 5'-AGATGAGCA TTGGCAGCGAG-3'; and GAPDH forward, 5'-GGAGCGAG ATCCCTCCAAAAT-3' and reverse, 5'-GGCTGTTGTCATAC TTCTCATGG-3'. Relative expression levels were calculated using the $2^{-\triangle \Delta C q}$ method (18) following normalization to the expression of GAPDH. 
Western blotting $(W B)$. Cells were lysed in NETN buffer (20 mM Tris- $\mathrm{HCl}$ at $\mathrm{pH}$ 8.0, $100 \mathrm{mM} \mathrm{NaCl}, 1 \mathrm{mM}$ EDTA, $0.5 \%$ Nonidet P-40) containing protease and phosphatase inhibitor cocktails (Thermo Fisher Scientific, Inc.). The lysate protein concentration was measured using the BCA protein assay kit (Pierce; Thermo Fisher Scientific, Inc.). After normalization to equal amounts, $10 \mu \mathrm{g}$ each protein sample was separated via $10 \%$ SDS-PAGE, transferred to PVDF membranes and blocked with 5\% non-fat milk diluted in 1X PBS supplemented with $0.5 \%$ Tween-20 (PBST) at room temperature for $1 \mathrm{~h}$. Then, the membranes were probed with the following primary antibodies at $4^{\circ} \mathrm{C}$ overnight: GTSE1 (cat. no. A302-425A; Bethyl Laboratories, Inc.; 1:1,000), SNAIL1 (cat. no. 3879; Cell Signaling Technology, Inc.; 1:1,000), Tubulin (cat. no. 66031-1-Ig; ProteinTech Group, Inc.; 1:5,000), Lamin B1 (cat. no. 66095-1-Ig; ProteinTech Group, Inc.; 1:1,000), anti-Flag tag (cat. no. F3165; Sigma-Aldrich; Merck KGaA; 1:3,000) and anti-human influenza hemagglutinin epitope (HA) tag (the amino acid sequence is YPYDVPDYA; cat. no. 3724; Cell Signaling Technology, Inc.; 1:1,000). The blots were then incubated with species-specific HRP-conjugated secondary antibodies (cat. no. W4011 for rabbit and cat. no. W4021 for mouse-originated primary antibodies; Promega Corporation; $1: 5,000)$ at room temperature for $1 \mathrm{~h}$, and the immunoreactive bands were visualized using an ECL reagent (cat. no. 6883; Cell Signaling Technology, Inc.). The primary antibodies were diluted in Primary Antibody Dilution Buffer (cat. no. P0023A; Beyotime Institute of Biotechnology), and the secondary antibodies were diluted in 1X PBST. Tubulin or Lamin B1 was used as a loading control. Semi-quantification of band densitometry was measured with ImageJ software (version 1.47; National Institutes of Health).

Plasmid construction and transfection. DNA fragments containing the coding sequence (CDS) of full-length human GTSE1 and SNAIL1 were amplified via nested PCR from a cDNA library of 293 T cells using the PrimeSTAR ${ }^{\circledR}$ Max DNA Polymerase (cat. no. R045A; Takara Bio, Inc.). The thermocycling conditions were as follows: Initial denaturation for $3 \mathrm{~min}$ at $98^{\circ} \mathrm{C}$, followed by 35 cycles of denaturation for $15 \mathrm{sec}$ at $98^{\circ} \mathrm{C}$, annealing for $5 \mathrm{sec}$ at $55^{\circ} \mathrm{C}$ and elongation for $30 \mathrm{sec}$ at $72^{\circ} \mathrm{C}$, followed by final extension for $5 \mathrm{~min}$ at $72^{\circ} \mathrm{C}$. The outer primer pairs used were as follows: GTSE1-outer-forward, 5'-GTTTAAATCCGTGCCGGAGG-3'; GTSE1-outer-reverse, 5'-AGGGCTGTTCTTTCAAGGCA-3'; and SNAIL1outer-forward, 5'-AGTGGTTCTTCTGCGCTACT-3'; SNAIL1outer-reverse, 5'-AGGCTGAAATAGCTGCCTGG-3'. The product of the first PCR was used as the template for the second PCR during which the sequences encoding Flag tag (5'-GATTACAAGGATGACGACGATAAG-3') or HA tag (5'-TACCCATACGATGTTCCAGATTACGCT-3') were added after the start codon to the $5^{\prime}$ end of the CDS region of the indicated protein. The product of the second PCR was then digested and ligated into the KpnI and EcoRI sites of the pcDNA3.1(+) vector (Invitrogen; Thermo Fisher Scientific, Inc.). Correct constructs were all confirmed via Sanger sequencing with the CMV-forward primer 5'-CGCAAATGG GCGGTAGGCGTG-3'.

Huh7 or PLC/PRF/5 cells were transfected with plasmids encoding GTSE1 and/or SNAIL1, and negative control
pcDNA3.1(+) plasmid using ViaFect ${ }^{\mathrm{TM}}$ transfection reagent (Promega Corporation) for $6 \mathrm{~h}$ at $37^{\circ} \mathrm{C}$ in a cell incubator following the manufacturer's protocol. For transfection in 6-well plates, a total of $2 \mu \mathrm{g}$ plasmid was used for each well. At $36 \mathrm{~h}$ post-transfection, the transfection efficiency was detected using WB.

RNA interference. A total of two targeting GTSE1 small interfering (si)RNA duplexes (siGTSE1\#1, 5'-GGATGTTCTCCCT GACAAA-3'; siGTSE1\#2,5'-GCCTACTCCTACAAATCAA-3') and the negative control (siN0000001) were obtained from Guangzhou RiboBio Co., Ltd. Huh7 cells were transfected with 100 nM siRNA using Lipofectamine ${ }^{\circledR}$ RNAiMax (Invitrogen; Thermo Fisher Scientific, Inc.) for $24 \mathrm{~h}$ at $37^{\circ} \mathrm{C}$ in a cell incubator, according to the manufacturer's protocol. At $72 \mathrm{~h}$ post-transfection, RNA interference was confirmed via RT-qPCR or WB.

Immunofluorescence. Cells (70\% confluence) were plated on chamber slides and fixed with $4 \%$ paraformaldehyde at room temperature for $5 \mathrm{~min}$. After fixation, cells were permeabilized with $0.1 \%$ Triton $\mathrm{X}-100$ for $5 \mathrm{~min}$. Then, cells were blocked with $10 \%$ FBS for $20 \mathrm{~min}$ at room temperature and incubated with the following primary antibodies at $4{ }^{\circ} \mathrm{C}$ overnight: Anti-Flag tag (cat. no. F3165; Sigma-Aldrich; Merck KGaA; 1:1,000) and anti-HA tag (cat. no. 3724; Cell Signaling Technology, Inc.; 1:500). The anti-Mouse IgG (H+L) Alexa Fluor ${ }^{\circledR} 488$ conjugate (cat. no. A-11001; Invitrogen; Thermo Fisher Scientific, Inc.; 1:200) or anti-Rabbit IgG (H+L) Alexa Fluor ${ }^{\circledR} 594$ conjugate secondary antibodies (cat. no. A-11012; Invitrogen; Thermo Fisher Scientific, Inc.; 1:200) were added and incubated in the dark for $60 \mathrm{~min}$ at room temperature. Nuclear staining was performed with $50 \mathrm{ng} / \mathrm{ml}$ DAPI (cat. no. D21490; Invitrogen; Thermo Fisher Scientific, Inc.) in the dark for $5 \mathrm{~min}$ at room temperature. The fluorescence signal was imaged using a Zeiss LSM710 confocal microscope at x400 magnification (Zeiss AG).

Luciferase reporter assay. A GTSE1 promoter region from $-1,500$ to the transcription start site (TSS) was amplified via PCR, which was performed as aforementioned, and inserted into the KpnI and HindIII sites of pGL3-Basic plasmid (Promega Corporation), hereafter named pGL3-GTSE1-PM. In total, three potential Smad binding elements (SBEs) containing the $5 \mathrm{bp}$ CAGAC motif were found in the aforementioned GTSE1 promoter region, locating at -35 to -39 , -714 to -718 and $-1,117$ to -1121 , respectively. Accordingly, three GTSE1 promoter constructs, deleting one of the potential SBEs, were prepared via a PCR-based method, and were named pGL3-GTSE1-DEL1, pGL3-GTSE1-DEL2 and pGL3-GTSE1-DEL3.

A total of $\sim 1 \times 10^{5}$ Huh7 cells were seeded in 12-well plates and cultured for $24 \mathrm{~h}$. Then, $1 \mu \mathrm{g}$ each of the GTSE1 promoter constructs and $0.05 \mu \mathrm{g}$ control pRL-TK (Promega Corporation) encoding Renilla luciferase were then co-transfected into cells using ViaFect ${ }^{\mathrm{TM}}$ transfection reagent (Promega Corporation) for $6 \mathrm{~h}$ at $37^{\circ} \mathrm{C}$ in a cell incubator, following the manufacturer's procedures. At $30 \mathrm{~h}$ post-transfection, quantification of firefly and Renilla luciferase activities of $\geq 3$ independent transfections were measured with the Dual Reporter Assay system (Promega Corporation) using an FB12 luminometer 
(Titertek-Berthold). The relative luciferase activities were calculated by normalizing the activity of the fluorescent luciferase with that of the internal standard Renilla luciferase.

CHX chase experiment. Huh7 cells were transiently transfected with GTSE1 encoding plasmid and the negative control plasmid, or GTSE1 targeting siRNAs and negative control siRNA. At $30 \mathrm{~h}$ post-transfection with plasmids or $72 \mathrm{~h}$ post-transfection with siRNAs, transfected cells were treated with $10 \mu \mathrm{g} / \mathrm{ml} \mathrm{CHX}$ at $37^{\circ} \mathrm{C}$ for an additional time period, including $0,0.5,1$ and $2 \mathrm{~h}$. Then, cells were lysed in NETN buffer, and the SNAIL1 protein turnover was detected via WB, performed as aforementioned, using antibodies against GTSE1, SNAIL1, HA tag and Tubulin. Tubulin was used as a loading control.

Subcellular fractionation. Nuclear and cytoplasmic extracts of Huh7 cells were prepared using NE-PER nuclear and cytoplasmic extraction reagents (Pierce; Thermo Fisher Scientific, Inc.) according to the supplier's protocol.

Statistical analysis. SPSS software version 20.0 (IBM Corp.) and GraphPad Prism 6 software (GraphPad Software, Inc.) were used to perform statistical analyses. Statistical differences between two groups were determined using an unpaired Student's t-test, whereas statistical differences between multiple groups were determined using a one-way ANOVA, followed by a Tukey's post hoc test. Each experiment was performed three times in triplicate. Unless otherwise indicated, all error bars indicate SD. All statistical tests were two-sided, and $\mathrm{P}<0.05$ was considered to indicate a statistically significant difference.

\section{Results}

GTSE1 negatively regulates the protein expression level of SNAIL1 in HCC cells. Our previous study reported that GTSE1 was involved in regulating the expression level of SNAIL1 protein and promoted the metastatic ability of HCC cells via EMT process (13). However, the exact mechanism via which GTSE1 regulates SNAIL1 expression is unknown. The current study first examined the relationship between GTSE1 and SNAIL1 expression in Huh7 and PLC/PRF/5 cells. Transfection of plasmids encoding HA-GTSE1 caused notable overexpression of GTSE1, which reduced the protein expression level of SNAIL1 in HCC cells (Figs. 1A and S1A). By contrast, knockdown of GTSE1 expression by two independent siRNAs, which notably reduced the protein expression of GTSE1, enhanced the expression of SNAIL1 in HCC cells (Figs. 1B and S1B). These results indicated that GTSE1 negatively regulates protein expression level of SNAIL1 in HCC cells.

GTSE1 increases SNAIL1 protein turnover via a proteasome-dependent pathway. Further investigations revealed that GTSE1 induced a decrease in SNAIL1 expression in a concentration-dependent manner, and the addition of the proteasome inhibitor MG-132 reversed the reduction in SNAIL1 expression (Fig. 2A). A CHX chase experiment was then conducted, and it was found that GTSE1 overexpression significantly promoted the degradation rate of SNAIL1 (Fig. 2B), while
A
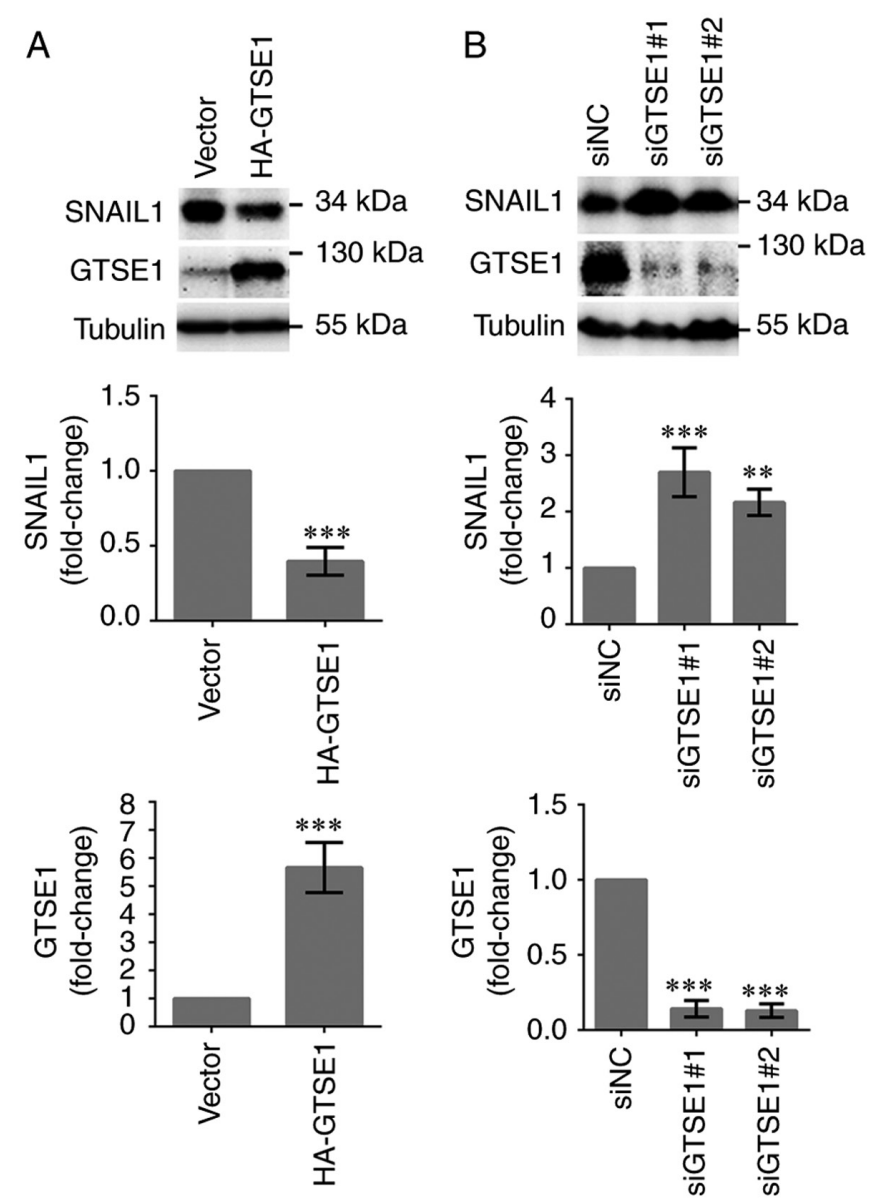

Figure 1. GTSE1 negatively regulates the protein expression level of SNAIL1 in hepatocellular carcinoma cells. Endogenous expression of SNAIL1 was detected via western blotting after GTSE1 (A) overexpression or (B) knockdown in Huh7 cells. Tubulin was used as a loading control. Semi-quantitative data of optical band densitometry are presented. The data are presented as the mean $\pm \mathrm{SD}(\mathrm{n}=3){ }^{* *} \mathrm{P}<0.01,{ }^{* * *} \mathrm{P}<0.001$ vs. Vector group or siNC group. GTSE1, $\mathrm{G}_{2}$ and $\mathrm{S}$ phase-expressed-1; SNAIL1, snail family transcriptional repressor 1; NC, negative control; si, small interfering RNA.

SNAIL1 protein turnover was delayed after GTSE1 knockdown in HCC cells (Fig. 2C). These results suggested that GTSE1 promoted the degradation of SNAIL1 protein via a proteasome-dependent pathway.

GTSE1 enhances the nuclear export of SNAIL1 in HCC cells. To determine whether GTSE1 expression influenced the subcellular localization of SNAIL1, HA-GTSE1 and Flag-SNAIL1 were co-expressed in HCC cells and an immunofluorescence assay was performed. As a major readout for this assay, exogenous Flag-SNAIL1 overexpression was firstly confirmed via WB in both Huh7 and PLC/PRF/5 cells (Fig. S2). The results demonstrated that Flag-SNAIL1 was mainly localized in the nucleus of HCC cells expressing Flag-SNAIL1 only, while its expression was detected in both the cytoplasm and the nucleus when HA-GTSE1 was co-expressed with Flag-SNAIL1 (Figs. 3A and B and S3A). Subcellular fractionation also demonstrated that GTSE1 overexpression enhanced the cytoplasmic localization of SNAIL1 (Fig. 3C). When the nuclear export inhibitor LMB was added, cytoplasmic retention of SNAIL1 was reduced, and Flag-SNAIL1 again displayed nuclear localization even 

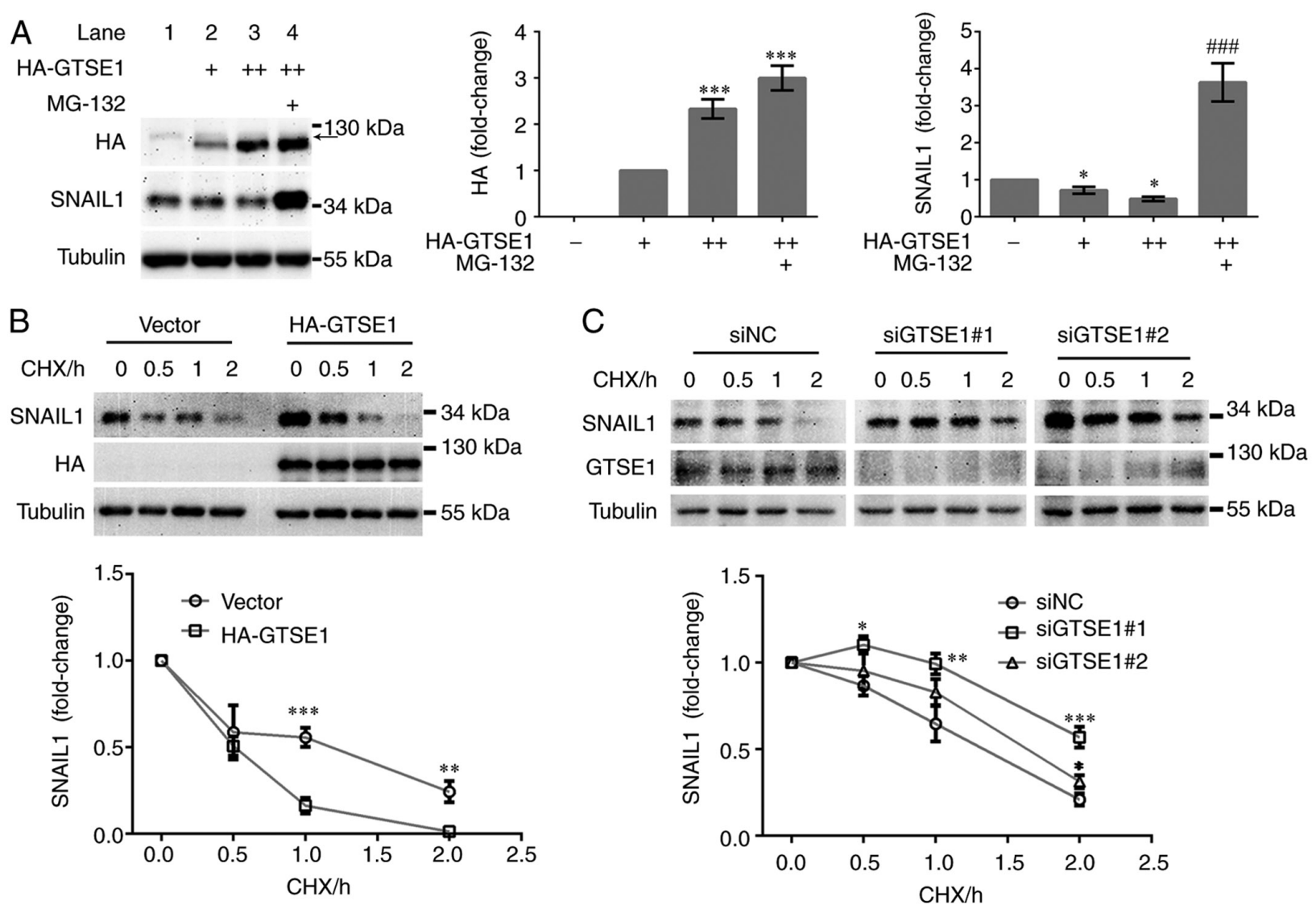

Figure 2. GTSE1 increases SNAIL1 protein turnover via a proteasome-dependent pathway. (A) Endogenous expression of SNAIL1 was detected via western blotting in Huh7 cells transfected with an increasing concentration of GTSE1 plasmid, and co-treatment using the proteasome inhibitor MG-132 (10 $\mu \mathrm{M}$ ) for an additional $12 \mathrm{~h}$ at $37^{\circ} \mathrm{C}$ before harvesting as indicated. The arrowhead indicates a non-specific band. Semi-quantitative data of optical band densitometry

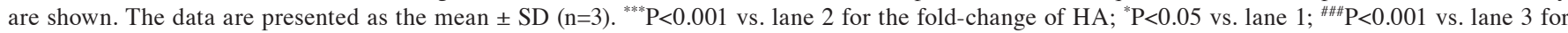
the fold-change of SNAIL1. Protein turnover of endogenous expression of SNAIL1 after GTSE1 (B) overexpression or (C) knockdown over the course of $2 \mathrm{~h}$ following the addition of $10 \mu \mathrm{g} / \mathrm{ml} \mathrm{CHX.} \mathrm{Tubulin} \mathrm{was} \mathrm{used} \mathrm{as} \mathrm{a} \mathrm{loading} \mathrm{control.} \mathrm{Images} \mathrm{are} \mathrm{representative} \mathrm{of} \mathrm{three} \mathrm{independent} \mathrm{experiments} \mathrm{and}$ fold-changes of SNAIL1 are shown below by densitometry, which was normalized to controls. The data are presented as the mean \pm SD ( $=3$ ). ${ }^{*}<0.05$, ${ }^{* *} \mathrm{P}<0.01,{ }^{* * *} \mathrm{P}<0.001$ vs. Vector group or siNC group at each time point. GTSE1, $\mathrm{G}_{2}$ and S phase-expressed-1; SNAIL1, snail family transcriptional repressor 1 ; $\mathrm{NC}$, negative control; si, small interfering RNA; CHX, cycloheximide; HA, human influenza hemagglutinin epitope.

under GTSE1 overexpression (Figs. 3D and E and S3B). These results indicated that GTSE1 induced the nuclear export of SNAIL1 in HCC cells.

TGF- $\beta$ I induces the expression of GTSE1 via SBEs in the promoter region. TGF- $\beta / \mathrm{Smad}$ signaling is able to induce EMT via upregulation of SNAIL1 expression in various human cancer types, including HCC (19). To determine whether GTSE1 was involved in TGF- $\beta$ signaling-mediated regulation of SNAIL1 expression, the expression of endogenous GTSE1 and SNAIL1 was first detected in the presence of TGF- $\beta$ I, a potent agonist of TGF- $\beta / \mathrm{Smad}$ signaling. Interestingly, an increasing expression level of GTSE1 but a decreasing expression level of SNAIL1 was observed after $24 \mathrm{~h}$ of treatment with a steadily growing concentration of TGF- $\beta$ I (Fig. 4A). Transcriptionally, TGF- $\beta$ I treatment induced the upregulation of only GTSE1 mRNA expression levels and affect not SNAIL1 mRNA expression levels in Huh7 cells (Fig. 4B).

TGF- $\beta$ I actives the transcription of gene expression via the binding of the downstream Smad transcription factors to SBE, the $5 \mathrm{bp} \mathrm{CAGAC}$ motif, in the promoter region of specific target genes (20). Therefore, the current study analyzed the sequence of the GTSE1 promoter region and found three potential SBEs located at the region from $-1,500$ to the TSS. Accordingly, luciferase reporter plasmids of GTSE1 promoter were created containing different SBE deletions as indicated in Fig. 4C. Under unstimulated conditions, reporter plasmids with the deletion of either SBE (GTSE1-DEL1, GTSE1-DEL2 or GTSE1-DEL3) showed comparable, but slightly lower transcriptional activity compared with the control plasmid (GTSE1-PM). After $24 \mathrm{~h}$ of TGF- $\beta$ I treatment, transcriptional activity of the GTSE1-PM and GTSE1-DEL3 plasmids increased nearly 5-fold compared with the unstimulated control. However, the transcriptional activity of either GTSE1-DEL1 or GTSE1-DEL2 plasmids showed no obvious induction even after TGF- $\beta$ I treatment compared with the unstimulated control (Fig. 4D). Moreover, knockdown of GTSE1 via transfection of siRNAs significantly reversed the decrease in SNAIL1 protein expression after TGF- $\beta$ I treatment, even though the mRNA level of SNAIL1 was not differentially changed (Fig. 4E and F). These results indicated that TGF- $\beta$ I treatment could upregulate the transcription of GTSE1 expression by transactivating the SBEs in GTSE1 promoter, and that the induced GTSE1 expression further promoted the degradation of SNAIL1 protein.

TGF- $\beta$ I-induced migratory ability of Huh7 cells is dependent on GTSE1 expression. In order to examine the biological 

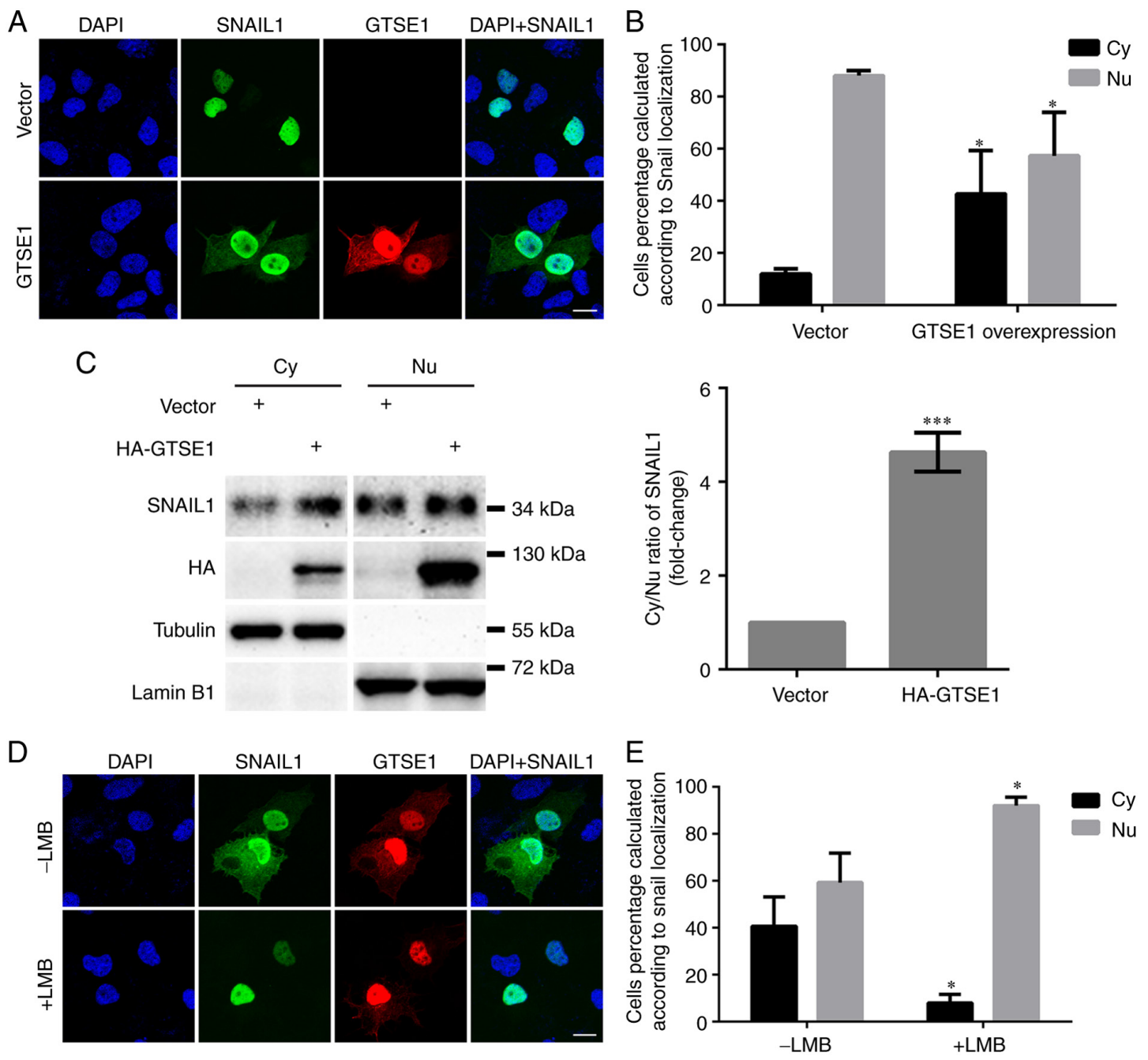

Figure 3. GTSE1 enhances the nuclear export of SNAIL1 in HCC cells. (A) Representative images of three independent immunofluorescence analysis assays showing the cellular localization of exogenously expressing Flag-SNAIL1 with or without HA-GTSE1 co-expression. Huh7 cells were co-transfected with plasmids encoding Flag-SNAIL1 and HA-GTSE1, and incubated for $24 \mathrm{~h}$. After cell fixation, Flag-SNAIL1 and HA-GTSE1 were detected via anti-Flag immunostaining (green staining) or anti-HA immunostaining (red staining), respectively. Nuclear staining was performed using DAPI (blue staining). Scale bar, $10 \mu \mathrm{m}$. (B) Quantification of the percentage of 50 exogenous Flag-SNAIL1-positive cells according to the subcellular localization of Flag-SNAIL1 for each group in (A) (50 Flag-SNAIL1-positive cells were quantified). "P<0.05 vs. Vector group. (C) SNAIL1 expression levels were increased in the cytoplasmic fraction upon GTSE1 overexpression. Huh7 cells were transfected with the indicated plasmids and incubated for $24 \mathrm{~h}$. Nuclear and cytoplasmic extracts were prepared by subcellular fractionation and subjected to immunoblotting analysis with the indicated antibodies. Tubulin and Lamin B1 were used as loading controls for the cytoplasmic or nuclear total proteins, respectively. Semi-quantitative data of the ratio of Cy/Nu SNAIL1 via densitometry are shown. The data are presented as the mean $\pm \mathrm{SD}(\mathrm{n}=3){ }^{* * * *} \mathrm{P}<0.001$ vs. Vector group. (D) Representative images of three independent immunofluorescence analysis assays showing the cellular localization of exogenously co-expressing Flag-SNAIL1 and HA-GTSE1 with or without LMB $(50 \mathrm{ng} / \mathrm{ml})$ treatment for $12 \mathrm{~h}$ at $37^{\circ} \mathrm{C}$. Scale bar, $10 \mu \mathrm{m}$. (E) Quantification of the percentage of 50 Flag-SNAIL1 and HA-GTSE1 double-positive cells according to the subcellular localization of Flag-SNAIL1 for each group in (D) (50 Flag-SNAIL1 and HA-GTSE1 double-positive cells were quantified). "P<0.05 vs. -LMB group. Cy, cytoplasm; Nu, nucleus; GTSE1, $\mathrm{G}_{2}$ and S phase-expressed-1; SNAIL1, snail family transcriptional repressor 1; LMB, leptomycin B; HA, human influenza hemagglutinin epitope.

processes altered by GTSE1 expression, the present study measured the cell proliferation rate, cell cycle distribution and migratory ability of Huh7 cells after GTSE1 overexpression or knockdown with or without TGF- $\beta$ I treatment. Cell proliferation and cell cycle were not significantly altered by TGF- $\beta$ I treatment after GTSE1 knockdown in Huh7 cells (Fig. S4A and B). However, TGF- $\beta$ I treatment caused an increase in the migrated cell number of Huh7 cells in the siNC group under TGF- $\beta$ I treatment conditions, regardless of the decrease of SNAIL1 expression, and there was no significant difference in the migration of the cells in the siGTSE1 group under TGF- $\beta$ I conditions compared with the control (Fig. 5A). Consistently, GTSE1 overexpression enhanced the migratory ability in Huh7 cells, despite the fact that increased SNAIL1 protein turnover was induced (Fig. 5B). There results suggested that the TGF- $\beta$ I-enhanced migratory ability was mainly due to the increased expression of GTSE1, but not the expression of SNAIL1, in Huh7 cells.

\section{Discussion}

The present study demonstrated that GTSE1 could promote the degradation of SNAIL1 protein in Huh7 HCC cells. Using immunofluorescence detection and subcellular fractionation assays, it was identified that GTSE1 overexpression enhanced the nuclear export of SNAIL1 protein. Moreover, in Huh7 cells, TGF- $\beta$ I treatment increased the mRNA transcription of GTSE1, but not that of SNAIL1, by transactivating the SBEs in the GTSE1 
A
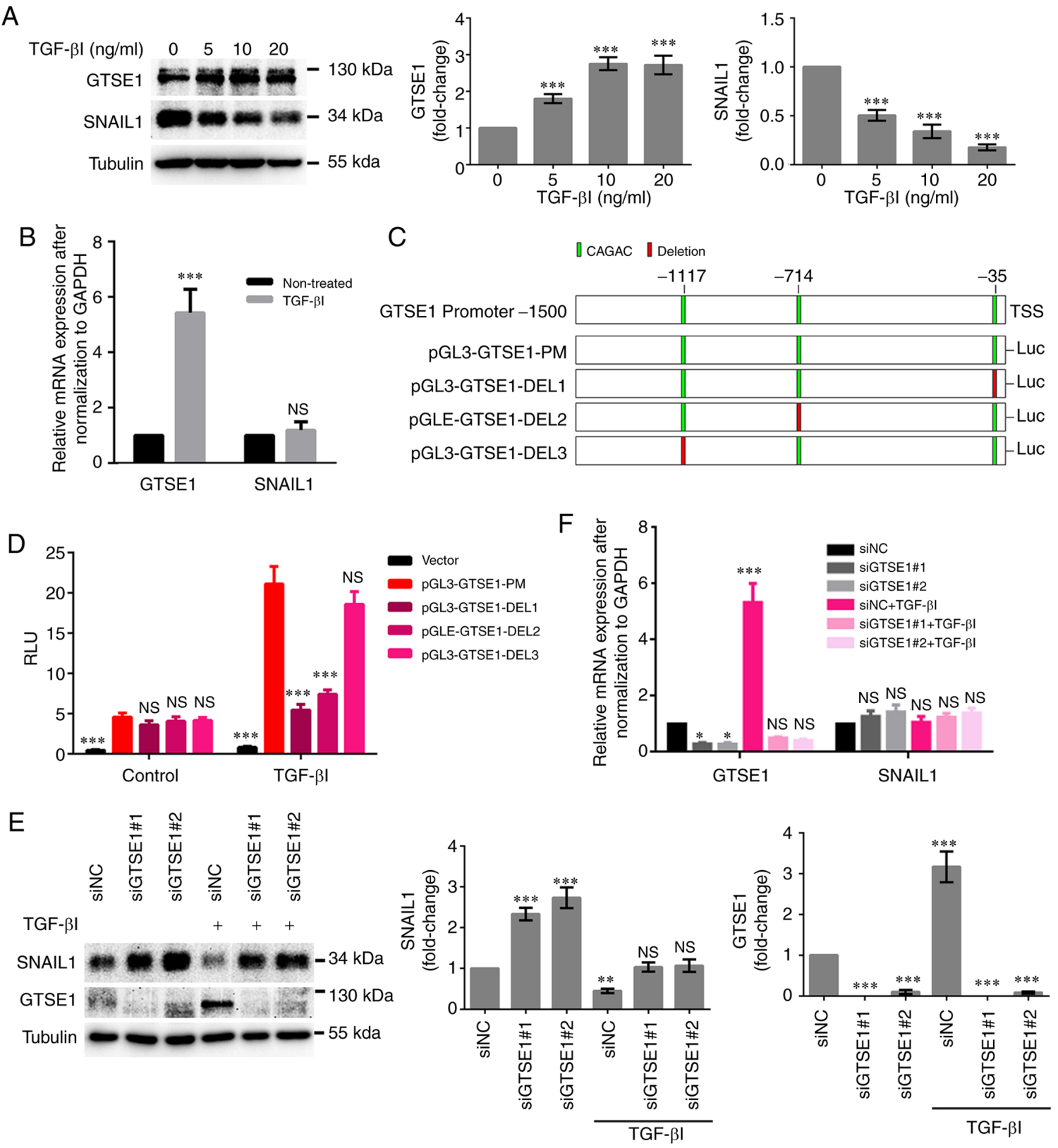

Figure 4. TGF- $\beta$ I induces the expression of GTSE1 via SBEs in the promoter region. (A) Endogenous protein expression levels of both GTSE1 and SNAIL1 were detected via western blotting with increasing concentrations of TGF- $\beta \mathrm{I}$ treatment for $24 \mathrm{~h}$ at $37^{\circ} \mathrm{C}$ in Huh7 cells. Semi-quantitative data of optical band densitometry are shown. The data are presented as the mean $\pm \mathrm{SD}(\mathrm{n}=3) .{ }^{* * * *} \mathrm{P}<0.001$ vs. control group. (B) GTSE1 and SNAIL1 mRNA expression levels were detected via RT-qPCR after TGF- $\beta$ I treatment $(20 \mathrm{ng} / \mathrm{ml})$ for $24 \mathrm{~h}$ at $37^{\circ} \mathrm{C}$ in Huh7 cells. GAPDH was used as an internal control. Data are expressed as the mean $\pm \mathrm{SD}(\mathrm{n}=3) .{ }^{* * *} \mathrm{P}<0.001$ and NS vs. non-treated group. (C) Schematic representation of pGL3-luc reporter constructs of the GTSE1 promoter (from $-1,500$ to TSS) with or without deletion of potential Smad binding elements (squares) as indicated. (D) RLU of different GTSE1 promoter constructs was determined using a luciferase reporter assay. Huh7 cells were co-transfected with the indicated pGL3-luc reporter constructs and Renilla luciferase. Then, 1 day after transfection, cells were treated with 0 or $20 \mathrm{ng} / \mathrm{ml}$ TGF- $\beta$ I for an additional $24 \mathrm{~h}$ at $37^{\circ} \mathrm{C}$. Data are expressed as the mean $\pm \mathrm{SD}(\mathrm{n}=3)$. ${ }^{* * *} \mathrm{P}<0.001$ and NS vs. pGL3-GTSE1-PM group. (E) Endogenous expression of SNAIL1 was detected via western blotting after GTSE1 depletion with or without TGF- $\beta$ I treatment in Huh7 cells. Semi-quantitative data of optical band densitometry are shown. The data are presented as the mean $\pm \mathrm{SD}(\mathrm{n}=3){ }^{* * *} \mathrm{P}<0.01,{ }^{* * * *} \mathrm{P}<0.001$ and NS, not significant vs. siNC group. (F) GTSE1 and SNAIL1 mRNA expression levels were detected via RT-qPCR after GTSE1 knockdown with or without TGF- $\beta$ I treatment in Huh7 cells. Data are expressed as the mean $\pm \mathrm{SD}(\mathrm{n}=3) .{ }^{*} \mathrm{P}<0.05,{ }^{* * * *} \mathrm{P}<0.001$ and NS vs. siNC group. NS, not significant; RLU, relative luciferase activity; GTSE1, $\mathrm{G}_{2}$ and S phase-expressed-1; SNAIL1, snail family transcriptional repressor 1; NC, negative control; si, small interfering RNA; TSS, transcription start site; DEL, deletion; PM, promoter.

promoter region, subsequently leading to the upregulation of GTSE1 protein expression and the downregulation of SNAIL1 protein expression. Therefore, the current study provides a novel mechanism via which GTSE1 affects the stability of SNAIL1 by regulating its subcellular localization in HCC cells.

The most important finding of the present study was that GTSE1 overexpression could promote the nuclear export of
SNAIL1 protein, thus leading to its enhanced degradation. The subcellular localization of SNAIL1 is one of the key factors regulating its stability $(11,12)$. SNAIL1 is degraded by proteasomes in the cytosol after nuclear export induced by GSK-3 $\beta$-dependent phosphorylation and $\beta$-Trcp-mediated ubiquitination (11). Other modulators, such as p21 (RAC1) activated kinase 1 (PAK1) and large tumor suppressor kinase 2 (Lats2), phosphorylate SNAIL1 
A
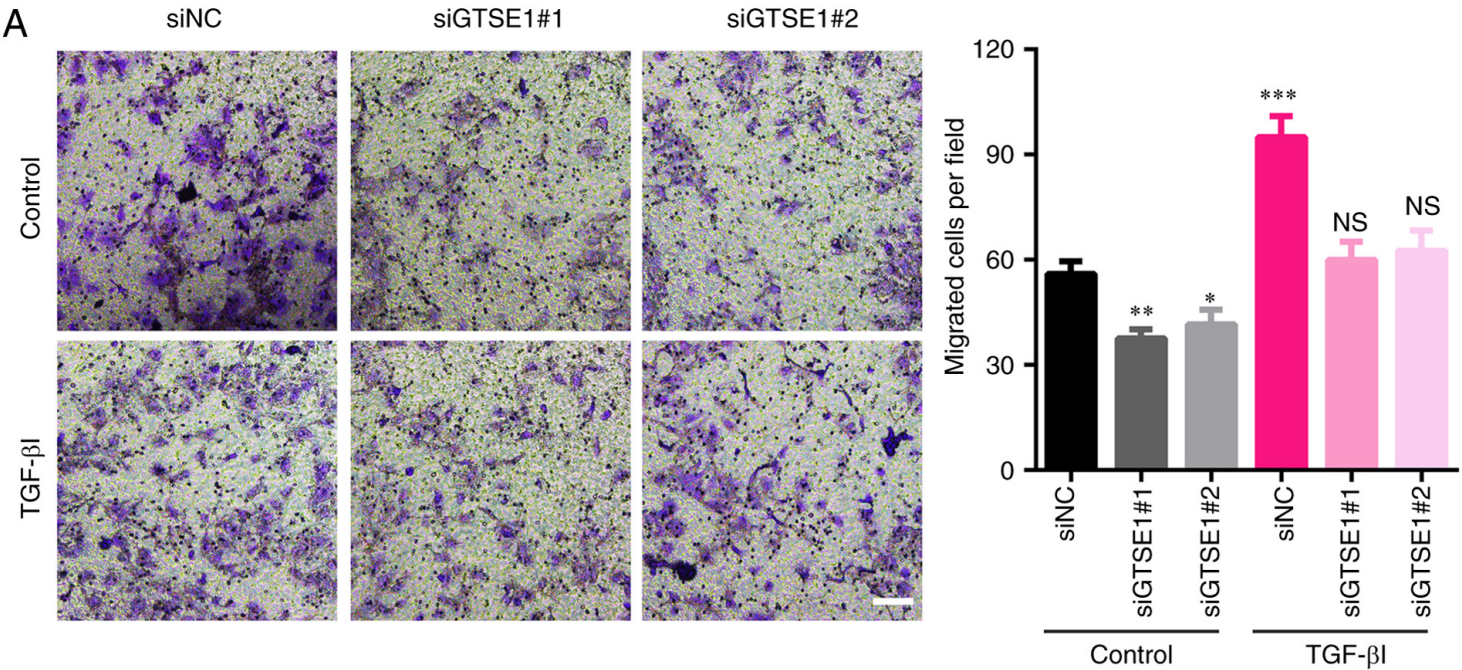

$\mathrm{B}$
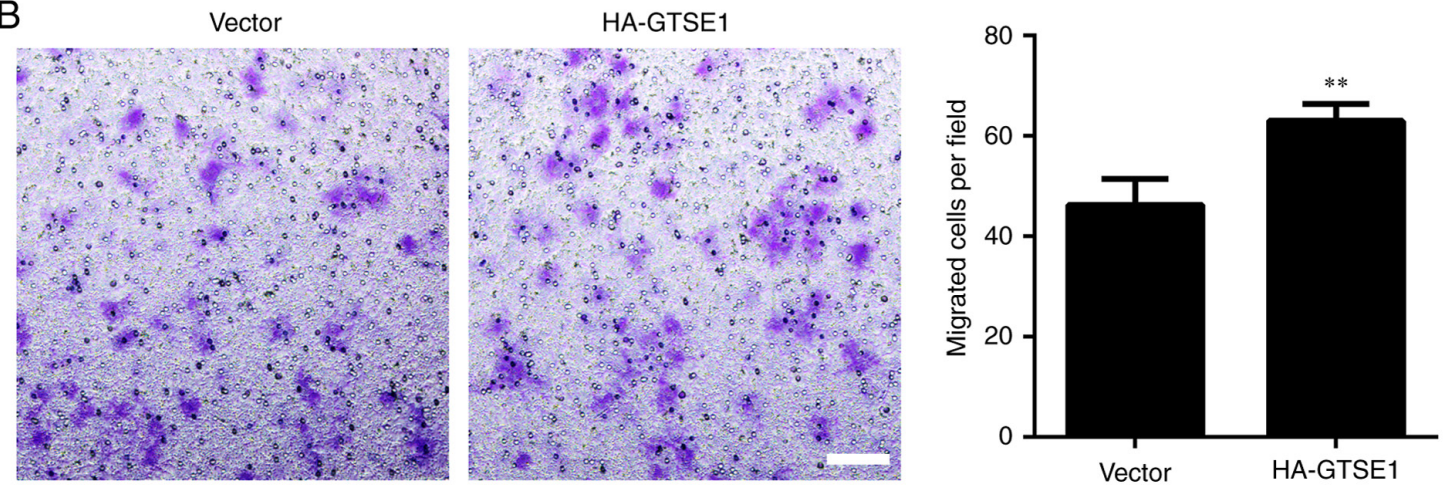

Figure 5. TGF- $\beta$ I-induced migratory ability of Huh7 cells is dependent on GTSE1 expression. (A) Transwell assay of Huh7 cells after GTSE1 knockdown with or without TGF- $\beta$ I treatment. Left panel: Representative images of three independent assays. Scale bar, $50 \mu \mathrm{m}$. Right panel: Quantification of the migrated cells per field for each group $(\mathrm{n}=3) .{ }^{*} \mathrm{P}<0.05,{ }^{* *} \mathrm{P}<0.01,{ }^{* * * *} \mathrm{P}<0.001$ and NS vs. siNC group. (B) Transwell assay of Huh7 cells with GTSE1 overexpression. Left panel: Representative images of three independent assays. Scale bar, $50 \mu \mathrm{m}$. Right panel: Quantification of the migrated cells per field for each group (n=3).

${ }^{* *} \mathrm{P}<0.01$ vs. Vector group. NS, not significant; GTSE1, $\mathrm{G}_{2}$ and $\mathrm{S}$ phase-expressed-1; NC, negative control; si, small interfering RNA.
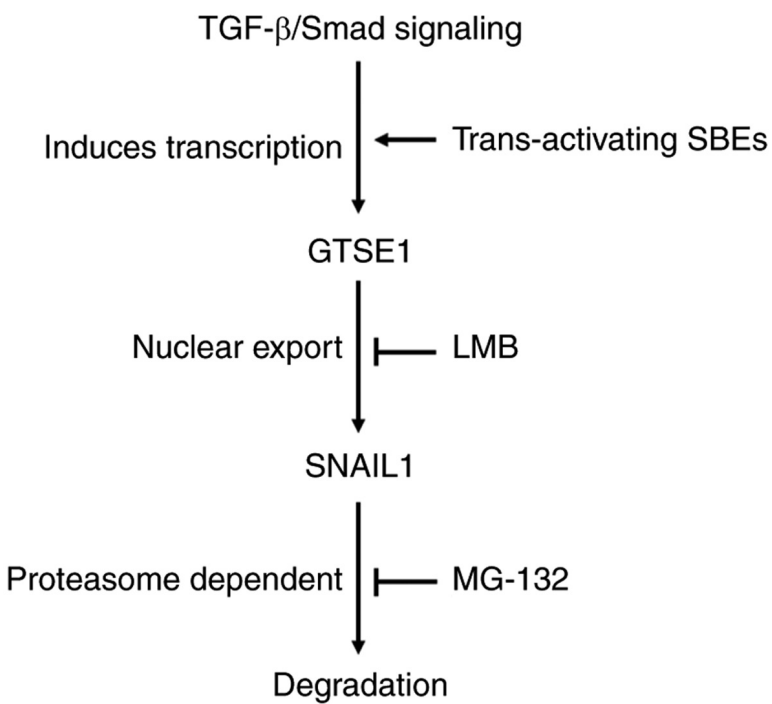

Figure 6. A schematic diagram depicting the TGF- $\beta /$ Smad-GTSE1-SNAIL1 signaling axis in Huh7 cells. GTSE1, $\mathrm{G}_{2}$ and $\mathrm{S}$ phase-expressed-1; SNAIL1, snail family transcriptional repressor 1 ; LMB, leptomycin B; SBEs, Smad binding elements.

at Ser246 or Thr203, respectively, acting to retain SNAIL1 in the nucleus, thereby enhancing its stability $(21,22)$. GTSE1 can function as a shuttle protein transporting proteins from the nucleus to the cytosol. For example, it has been reported that GTSE1 could promote p53 translocation out of the nucleus via physical interaction with its C-terminal regulatory domain in cells after $\mathrm{G}_{2}$ checkpoint recovery $(23,24)$. These aforementioned studies provide a theoretical basis that GTSE1 regulates the stability of SNAIL1 by changing its subcellular localization, although the present study could not identify a direct interaction between these two proteins using an immunoprecipitation assay (data not shown). Therefore, the underlying mechanism requires further investigation.

Another interesting finding of the present study was that TGF- $\beta$ I treatment increased both the mRNA and protein expression levels of GTSE1 and decreased the protein level of SNAIL1 without affecting its mRNA level. TGF- $\beta$ is a cytokine with pleiotropic functions that serves either a tumor suppressor or tumor promoter role in vivo, depending on the stage of tumorigenesis $(25,26)$. TGF- $\beta$ signaling also serves a central role in triggering EMT, which further provides cancer cells with enhanced motility and survival (19). As an important EMT inducer, SNAIL1 is upregulated by TGF- $\beta$ signaling and promotes liver tumorigenesis during the early stages in a mouse model of oncogene-driven liver tumorigenesis (27). However, in another in vitro report using Huh7 cells, the same HCC cell model used in the current study, TGF- $\beta$ stimulation 
had no effect on SNAIL1 mRNA expression and could not induce an EMT expression profile in Sox9- Huh7 cells, which was consistent with the current results (28). Furthermore, to the best of our knowledge, the present study was the first to report that GTSE1 was one of the downstream targets of TGF- $\beta /$ Smad signaling, and that the TGF- $\beta$-induced GTSE1 may promote the degradation of SNAIL1 by interfering with its subcellular localization in HCC cells (Fig. 6). However, the multifunctional role of TGF- $\beta$ in HCC development and progression requires further clarification.

Nonetheless, there were some limitations to the present study. No clinical samples were analyzed, and tissue samples could be used to further verify the findings in this study. In addition, since GTSE1 did not show a direct interaction with SNAIL1, the mediators that facilitate the nuclear export of SNAIL1 by GTSE1 overexpression await discovery. It is possible that GTSE1 overexpression affects the enzyme activity or localization of regulators upstream of SNAIL1, such as GSK-3 $\beta$, PAK1 and Lats, via an unknown mechanism, and this should be investigated.

In conclusion, the present study demonstrated that GTSE1 overexpression promoted the degradation of SNAIL1 protein by facilitating the nuclear export of SNAIL1. TGF- $\beta / \mathrm{Smad}$ signaling increased both the mRNA and protein expression levels of GTSE1, possibly leading to a subsequent decrease in SNAIL1 protein expression without affecting its mRNA transcription in Huh7 cells. Therefore, the present study provides a novel mechanism via which GTSE1 affects the stability of SNAIL1 by regulating its subcellular localization in HCC cells.

\section{Acknowledgements}

Not applicable.

\section{Funding}

This study was supported in part by the Natural Science Foundation of Guangdong Province (grant no. 2018A030313592), the National Natural Science Foundation of China (grant no. 81902397) and Fundamental Research Funds for the Central Universities (grant no. 20ykpy26).

\section{Availability of data and materials}

The datasets used and/or analyzed during the current study are available from the corresponding author on reasonable request.

\section{Authors' contributions}

YFL and YHH conceived the entire project. SSL and DMC carried out most of the experimental work. JX and LBC helped construct the luciferase reporter plasmids, performed the reporter assay and analyzed the data. HW and JLW helped with the acquisition and analysis of the confocal images of the immunofluorescence assays. YFL, YHH, SSL and DMC analyzed and interpreted most of the data. SSL, DMC, YHH and YFL confirmed the authenticity of all the raw data. YFL, SSL and YHH wrote the manuscript. SSL, JX and YFL revised the manuscript. YFL supervised the project and provided funding. All authors read and approved the final manuscript.

\section{Ethics approval and consent to participate}

Not applicable.

\section{Patient consent for publication}

Not applicable.

\section{Competing interests}

The authors declare that they have no competing interests.

\section{References}

1. Bray F, Ferlay J, Soerjomataram I, Siegel RL, Torre LA and Jemal A: Global cancer statistics 2018: GLOBOCAN estimates of incidence and mortality worldwide for 36 cancers in 185 countries. CA Cancer J Clin 68: 394-424, 2018.

2. Bruix J, Reig M and Sherman M: Evidence-based diagnosis, staging, and treatment of patients with hepatocellular carcinoma. Gastroenterology 150: 835-853, 2016.

3. Forner A, Reig M and Bruix J: Hepatocellular carcinoma. Lancet 391: 1301-1314, 2018.

4. Nieto MA: The snail superfamily of zinc-finger transcription factors. Nat Rev Mol Cell Biol 3: 155-166, 2002.

5. Domínguez D, Montserrat-Sentís B, Virgós-Soler A, Guaita S, Grueso J, Porta M, Puig I, Baulida J, Francí C and García de Herreros A: Phosphorylation regulates the subcellular location and activity of the snail transcriptional repressor. Mol Cell Biol 23: 5078-5089, 2003.

6. Wang Y, Shi J, Chai K, Ying X and Zhou BP: The role of snail in EMT and tumorigenesis. Curr Cancer Drug Targets 13: 963-972, 2013.

7. Kurrey NK, Jalgaonkar SP, Joglekar AV, Ghanate AD, Chaskar PD, Doiphode RY and Bapat SA: Snail and slug mediate radioresistance and chemoresistance by antagonizing p53-mediated apoptosis and acquiring a stem-like phenotype in ovarian cancer cells. Stem Cells 27: 2059-2068, 2009.

8. Moody SE, Perez D, Pan TC, Sarkisian CJ, Portocarrero CP, Sterner CJ, Notorfrancesco KL, Cardiff RD and Chodosh LA: The transcriptional repressor Snail promotes mammary tumor recurrence. Cancer Cell 8: 197-209, 2005.

9. Vincent T, Neve EP, Johnson JR, Kukalev A, Rojo F, Albanell J, Pietras K, Virtanen I, Philipson L, Leopold PL, et al: A SNAIL1-SMAD3/4 transcriptional repressor complex promotes TGF-beta mediated epithelial-mesenchymal transition. Nat Cell Biol 11: 943-950, 2009.

10. Ruan HB, Nie Y and Yang X: Regulation of protein degradation by O-GlcNAcylation: Crosstalk with ubiquitination. Mol Cell Proteomics 12: 3489-3497, 2013.

11. Zhou BP, Deng J, Xia W, Xu J, Li YM, Gunduz M and Hung MC: Dual regulation of Snail by GSK-3beta-mediated phosphorylation in control of epithelial-mesenchymal transition. Nat Cell Biol 6: 931-940, 2004.

12. Muqbil I, Wu J, Aboukameel A, Mohammad RM and Azmi AS: Snail nuclear transport: The gateways regulating epithelial-to-mesenchymal transition? Semin Cancer Biol 27: 39-45, 2014.

13. Wu X, Wang H, Lian Y, Chen L, Gu L, Wang J, Huang Y, Deng M, Gao Z and Huang Y: GTSE1 promotes cell migration and invasion by regulating EMT in hepatocellular carcinoma and is associated with poor prognosis. Sci Rep 7: 5129, 2017.

14. Monte M, Benetti R, Collavin L, Marchionni L, Del Sal G and Schneider C: hGTSE-1 expression stimulates cytoplasmic localization of p53. J Biol Chem 279: 11744-11752, 2004.

15. Scolz M, Widlund PO, Piazza S, Bublik DR, Reber S, Peche LY, Ciani Y, Hubner N, Isokane M, Monte M, et al: GTSE1 is a microtubule plus-end tracking protein that regulates EB1-dependent cell migration. PLoS One 7: e51259, 2012.

16. Subhash VV, Tan SH, Tan WL, Yeo MS, Xie C, Wong FY, Kiat ZY, Lim R and Yong WP: GTSE1 expression represses apoptotic signaling and confers cisplatin resistance in gastric cancer cells. BMC Cancer 15: 550, 2015. 
17. Lian YF, Huang YL, Zhang YJ, Chen DM, Wang JL, Wei H, Bi YH, Jiang ZW, Li P, Chen MS, et al: CACYBP enhances cytoplasmic retention of P27Kip1 to promote hepatocellular carcinoma progression in the absence of RNF41 mediated degradation. Theranostics 9: 8392-8408, 2019.

18. Livak KJ and Schmittgen TD: Analysis of relative gene expression data using real-time quantitative PCR and the 2(-Delta Delta C(T)) method. Methods 25: 402-408, 2001.

19. Chen J, Ding ZY, Li S, Liu S, Xiao C, Li Z, Zhang BX, Chen XP and Yang $X$ : Targeting transforming growth factor- $\beta$ signaling for enhanced cancer chemotherapy. Theranostics 11: 1345-1363, 2021.

20. Martin-Malpartida P, Batet M, Kaczmarska Z, Freier R, Gomes T, Aragón E, Zou Y, Wang Q, Xi Q, Ruiz L, et al: Structural basis for genome wide recognition of 5-bp GC motifs by SMAD transcription factors. Nat Commun 8: 2070, 2017.

21. Yang Z, Rayala S, Nguyen D, Vadlamudi RK, Chen S and Kumar R: Pak1 phosphorylation of snail, a master regulator of epithelial-to-mesenchyme transition, modulates snail's subcellular localization and functions. Cancer Res 65: 3179-3184, 2005.

22. Zhang K, Rodriguez-Aznar E, Yabuta N, Owen RJ, Mingot JM, Nojima H, Nieto MA and Longmore GD: Lats2 kinase potentiates Snaill activity by promoting nuclear retention upon phosphorylation. EMBO J 31: 29-43, 2012.
23. Monte M, Benetti R, Buscemi G, Sandy P, Del Sal G and Schneider C: The cell cycle-regulated protein human GTSE-1 controls DNA damage-induced apoptosis by affecting p53 function. J Biol Chem 278: 30356-30364, 2003.

24. Liu XS, Li H, Song B and Liu X: Polo-like kinase 1 phosphorylation of G2 and S-phase-expressed 1 protein is essential for p53 inactivation during $\mathrm{G} 2$ checkpoint recovery. EMBO Rep 11: 626-632, 2010.

25. Bierie B and Moses HL: Tumour microenvironment: TGFbeta: the molecular Jekyll and Hyde of cancer. Nat Rev Cancer 6: 506-520, 2006.

26. Massagué J: TGF $\beta$ signalling in context. Nat Rev Mol Cell Biol 13: 616-630, 2012.

27. Moon H, Ju HL, Chung SI, Cho KJ, Eun JW, Nam SW, Han KH, Calvisi DF and Ro SW: Transforming growth factor- $\beta$ promotes liver tumorigenesis in mice via up-regulation of snail. Gastroenterology 153: 1378-1391.e6, 2017.

28. Kawai T, Yasuchika K, Ishii T, Miyauchi Y,Kojima H, Yamaoka R, Katayama H, Yoshitoshi EY, Ogiso S, Kita S, et al: SOX9 is a novel cancer stem cell marker surrogated by osteopontin in human hepatocellular carcinoma. Sci Rep 6: 30489, 2016.

(i) (8) $\ominus$ This work is licensed under a Creative Commons cc) Attribution-NonCommercial-NoDerivatives 4.0 International (CC BY-NC-ND 4.0) License. 